\title{
A Case of Kawasaki Disease with Initial Presentation of Arthritis and Icterus
}

Nilüfer GÖKNAR ${ }^{1}$, Ayşegül Doğan DEMIR², Yasemin ATAMAN², Selman GÖKALP³, Faruk ÖKTEM1', Özgür KASAPÇOPUR

${ }^{1}$ Department of Pediatric Nephrology, Bezmialem Vakıf University School of Medicine, İstanbul, Turkey

${ }^{2}$ Department of Pediatrics, Bezmialem Vakıf University School of Medicine, İstanbul, Turkey

${ }^{3}$ Department of Pediatrics Cardiology, Bezmialem Vakıf University School of Medicine, İstanbul, Turkey

${ }^{4}$ Department of Rheumatology, İstanbul University School of Medicine, İstanbul, Turkey

\section{ABSTRACT}

Kawasaki disease is a systemic vasculitis that generally affects infants and children. Its diagnosis depends on clinical findings. Early diagnosis and treatment are important for the prevention of coronary dilatations. Late diagnosis due to different presentations can result in diagnostic difficulties and increased mortality. In this case report, we present the case of a 3-year-old boy who was admitted to our clinic with arthritis and icterus and who was diagnosed with Kawasaki disease.

Keywords: Kawasaki disease, arthritis, gallbladder hydrops

\section{Introduction}

Kawasaki disease (KD), which is also known as mucocutaneous lymph node syndrome, is a childhood vasculitis that presents with coronary disorders (1). It is characterized by persistent fever that lasts longer than 5 days, mucosal inflammation (erythema in the mouth and pharynx, strawberry tongue, and red and cracked lips), bilateral non-purulent conjunctivitis, erythematous rash, changes in the extremities (edema or erythema in the hands and feet and denudation in the fingertips), and cervical lymphadenopathy (unilateral and with the diameter of at least $1,5 \mathrm{~cm})(2)$. Although rare, it can present with aseptic meningitis, uveitis, urethritis, impaired liver functions, diarrhea, vomiting, gallbladder hydrops, and arthritis (3).

Systemic juvenile idiopathic arthritis (JIA) is characterized by prolonged fever that peaks once or twice a day, erythematous rash, lymphadenomegaly, and arthritis (4). While pericardial effusion is one of its frequent complications, myocarditis is seen rarely. Coronary artery dilatation has also been reported in the cases with systemic JIA (5). Clinical and laboratory findings of $\mathrm{KD}$ can be similar to those of JIA in some cases, which can lead to difficulty in the establishment of diagnosis (6).

Hepatobiliary involvement is considered a minor clinical finding in KD. Hepatobiliary dysfunction can cause damage in the bile ducts and gallbladder hydrops; however, jaundice and increased bilirubin levels are rarely encountered. Hepatomegaly and elevated aminotransferase were reported in $14,5 \%$ and $20 \%-30 \%$ cases, respectively (7-9). In this study, we present a case with fever accompanied by icterus and arthritis and with a differential diagnosis of JIA and cholangitis.

\section{Case Presentation}

A 3-year-old male patient was admitted to our clinic due to the complaints of fever lasting for 3 days, rash, icterus, and swollen and painful joints. His medical history revealed that he had been given ceftriaxone therapy by another center when his cough had begun and icterus and other complaints had occurred on the second day of the therapy. The patient was hospitalized due to the pre-diagnosis of systemic JIA and cholangitis. In his physical examination, he appeared tired and 


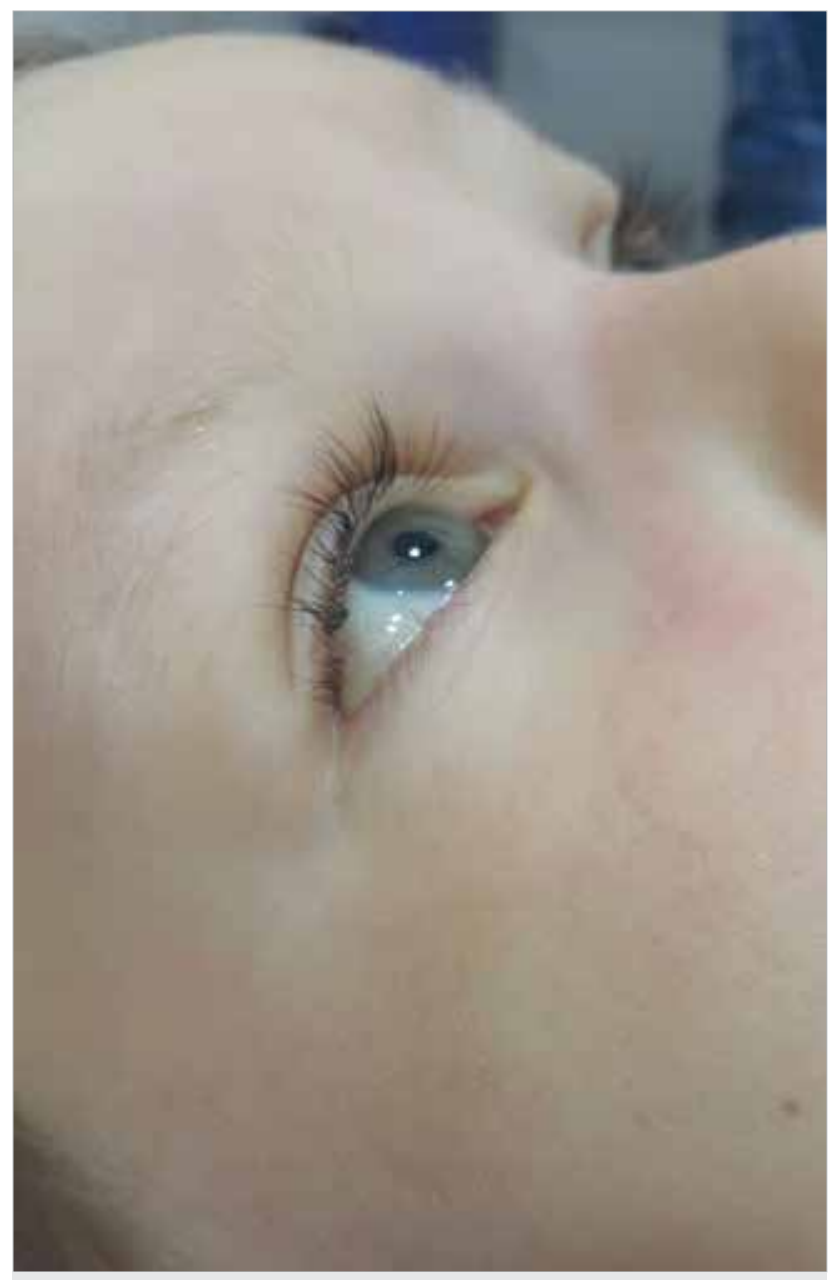

Picture 1. . Icteric appearance in conjunctivitis

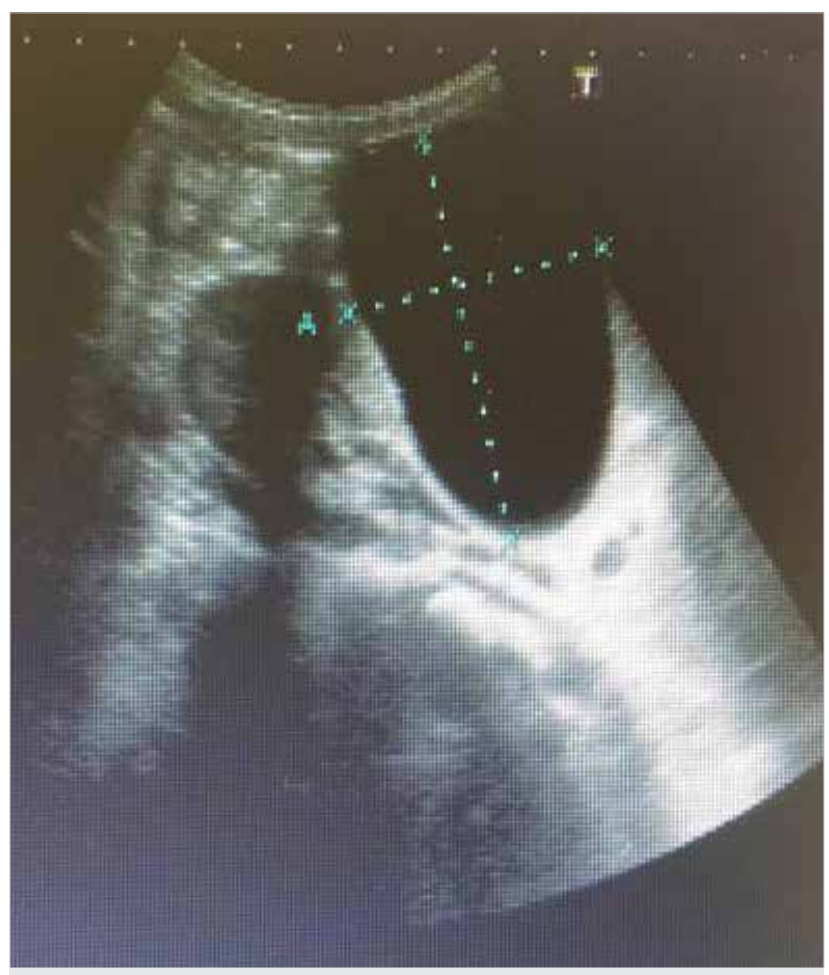

Picture 3. Hydrops in the gallbladder

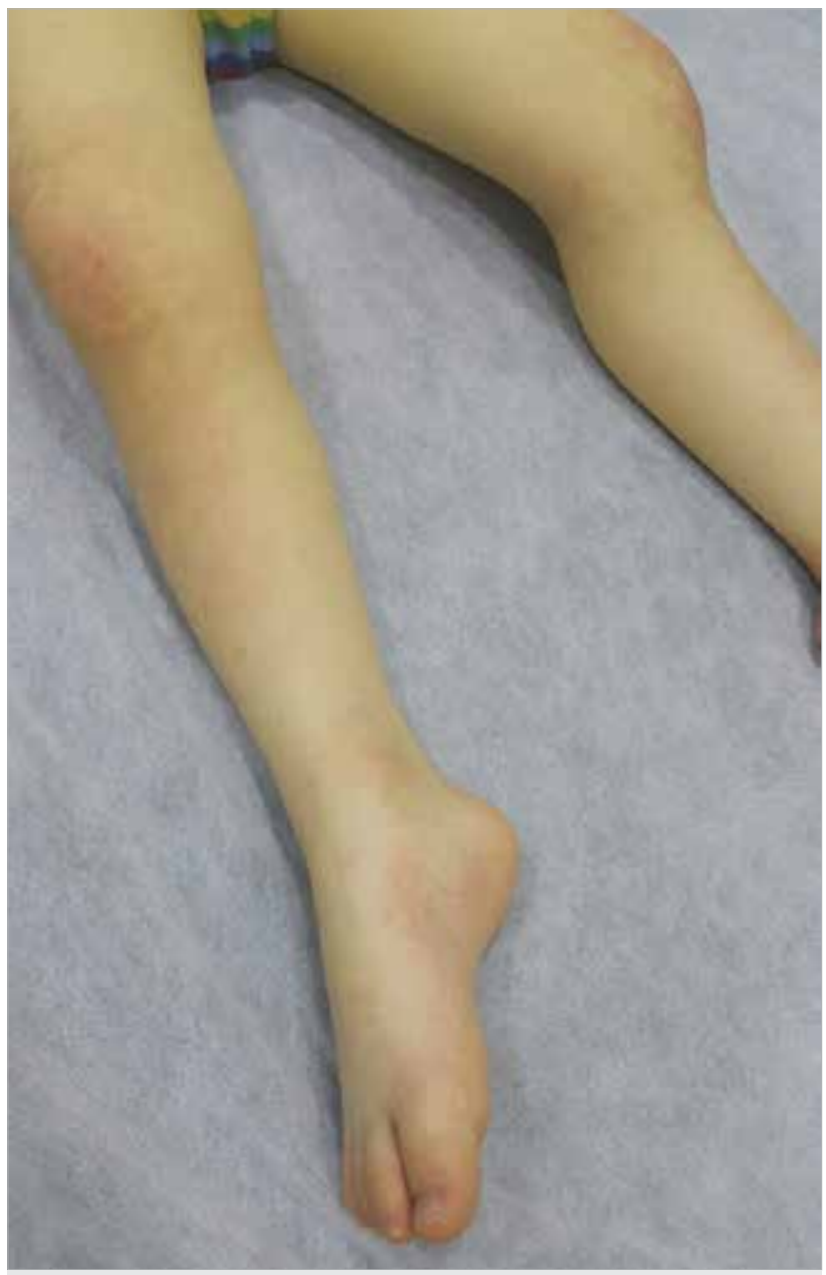

Picture 2. Swelling and redness in both knees

icteric. His axillary temperature was $38,5^{\circ} \mathrm{C}$. His conjunctivas were bilaterally hyperemic and icteric; his lips were hyperemic and crusted; his tongue was red and strawberry-like; and his distal and proximal interphalangeal joints in the lower and upper limbs and both knees were painful, red, and swollen. The palms and soles of the patient were erythematous and edematous (Pictures 1 and 2). His respiratory sounds were natural and the results of the cardiovascular system examination were normal. No lymphadenomegaly was detected. The liver was $1,5 \mathrm{~cm}$ and palpable. His fever was persistent. The results of the blood analysis were as follows: white blood cell (WBC): 24300/uL, hemoglobin ( $\mathrm{Hb}): 11.4 \mathrm{~g} / \mathrm{dL}$, platelet: 360000/uL, C-reactive protein (CRP): $20,9 \mathrm{mg} / \mathrm{dL}$, aspartate aminotransferase (AST): $149 \mathrm{U} / \mathrm{L}$, alanine aminotransferase (ALT): $150 \mathrm{U} / \mathrm{L}$, gamma-glutamyltransferase (GGT): 310 U/L, total bilirubin: $7,41 \mathrm{mg} / \mathrm{dL}$, direct bilirubin: $6,15 \mathrm{mg} /$ $\mathrm{dL}$, and albumin: 2,3 g/dL. All abdominal ultrasonographic (USG) examination results were evaluated to be normal. Blood culture, throat culture, and urinary culture were collected and no growth was observed. On the sixth day, fever continued and transthoracic echocardiographic examination revealed coronary artery dilatation (LMCA: $4 \mathrm{~mm}$ [z score: 3,26], LAD: $3 \mathrm{~mm}$ [z score: 2,97], RCA: $3 \mathrm{~mm}$ [z score: 
$2,47])$. Acetylsalicylic acid $(80 \mathrm{mg} / \mathrm{kg} /$ day $)$ and intravenous immunoglobulin (IVIG) $(2 \mathrm{~g} / \mathrm{kg})$ treatment was initiated. When abdominal USG was repeated, hydrops was observed in the gallbladder (Picture 3). Due to presence of icterus and hydropic sac, steroid $(2 \mathrm{mg} / \mathrm{kg} /$ day $)$ was added to his therapy. His fever, arthritis, and icterus were observed to be regressed in the follow-up. In the echocardiographic examination performed 2 months later, coronary dilatation was found to be completely regressed. Written and verbal informed consent was received from his parents.

\section{Discussion}

The diagnosis of KD is established with the presence of fever lasting for longer than 5 days and four of five diagnostic accompanying criteria. If not diagnosed and treated early, serious cardiovascular complications develop in $25 \%$ of cases. Within the first 10 days, the risk of coronary artery lesion can be decreased to $5 \%$ with the administration of aspirin and IVIG (8). In its atypical and incomplete forms, the risk of coronary artery disease is increased due to difficulties in diagnosis and late initiation of treatment. In our case, the complaints of fever, rash, conjunctivitis, edema in the hands and feet, and mucosal inflammation were accompanied by arthritis and icterus. Systemic JIA, cholangitis, and KD were considered as pre-diagnoses.

$\mathrm{KD}$ and systemic JIA with prolonged fever, rash, and lymphadenopathy and with high mortality are two important diseases considered in differential diagnosis. High CRP, leukocytosis, thrombocytosis, hypoalbuminemia, and anemia can be seen in both diseases. Moreover, coronary artery dilatation was reported in some systemic JIA cases. It was reported that some KD patients respond slightly to IVIG, but respond better to steroids $(6,9)$. In our case, systemic JIA was considered in differential diagnosis due to arthritis and increased acute phase reactants accompanying high fever; however, it was then ruled out because fever did not peak unlike systemic JIA, it was persistent, its history of onset was shorter than that in systemic JIA (6 weeks in the diagnostic criteria of systemic JIA), and it coexisted with conjunctivitis and mucosal inflammation findings and gallbladder hydrops.

Gallbladder hydrops was reported in a case of KD in 1970s. With increasing use of USG, its incidence is now known to be between $5 \%$ and $20 \%$. Although its pathogenesis is not exactly known, it is thought to be the inflammation of the gallbladder wall and vasculitis. It is usually seen in the first 2 weeks (10). It has been shown that patients particularly with gallbladder hydrops have higher neutrophil and CRP levels. In cases with hydrops, IVİG resistance is more frequent. Hydrops can occur before the development of other $\mathrm{KD}$ criteria. The risk of coronary artery aneurysm is increased in these cases. In some cases, icterus is included in differential diagnosis with acute surgical abdomen due to fever and abdominal pain (11). In our case, diagnosis was established considering the existence of four diag- nostic criteria (mucosal inflammation, conjunctivitis, changes in distal extremities, and rash) accompanying fever lasting for more than 5 days and coronary artery dilatation in echocardiographic examination. The detection of coronary artery dilatation on the sixth day and visualization of hydropic sac in USG made the differential diagnosis easier. In recent years, the use of steroid therapy is recommended for the findings of gastrointestinal system in KD (10). In our case, IVIG and steroid therapy were used together and it was observed that both gastrointestinal system findings and cardiac findings of the patient were regressed in the follow-up. The patient is still followed up without any problem.

\section{Conclusion}

Because this patient applied with arthritis and icterus, systemic JIA and KD were included in the differential diagnosis, and this made the establishment of diagnosis difficult. We aimed to demonstrate that patients can apply with different findings although to diagnose $\mathrm{KD}$ is generally not so difficult owing to its typical clinical findings.

Informed Consent: Written informed consent was obtained from patients who participated in this study.

Peer-review: Externally peer-reviewed.

Author Contributions: Concept - N.G., A.D.D.; Design - N.G., Y.A.; Supervision - Y.A., N.G.; Funding - A.D.D., S.G.; Materials - S.G., F.Ö.; Data Collection and/or Processing - F.Ö., S.G.; Analysis and/or Interpretation - N.G., A.D.D.; Literature Review - Y.A.; Writing - N.G.; Critical Review - F.Ö.

Conflict of Interest: No conflict of interest was declared by the authors.

Financial Disclosure: The authors declared that this study has received no financial support.

\section{References}

1. Yamamoto LG. Kawasaki disease. Pediatr Emerg Care 2003; 19: 422-4. [CrossRef]

2. Rashid AK, Kamal SM, Ashrafuzzaman M, Mustafa KG. Kawasaki disease and its treatment-an update. Curr Rheumatol Rev 2014; 10: 109-16. [CrossRef]

3. Kuo HC, Yang KD, Chang WC, Ger LP, Hsieh KS. Kawasaki disease: an update on diagnosis and treatment. Pediatr Neonatol 2012; 53 : 4-11. [CrossRef]

4. Pettyre RE, Southwood TR, Manners P, Baum J, Glass DN, Goldenberg J et al. International League of Associations for Rheumatology classification of juvenile idiopathic arthritis: second revision, Edmonton, 2001. J Rheumatol 2004; 31: 390-2.

5. Binstadt BA, Levine JC, Nigrovic PA, Gauvreau K, Dedeoglu F, Fuhlbrigge $\mathrm{RC}$ et al. Coronary artery dilatation among patients presenting with systemic-onset juvenile idiopathic arthritis. Pediatrics 2005; 116: e89-93.

6. Takahara T, Shimizu M, Nakagishi Y, Kinjo N, Yachie A. Serum IL18 as a potential specific marker for differentiating systemic juvenile idiopathic arthritis from incomplete Kawasaki disease. Rheumatol Int 2015; 35: 81-4. [CrossRef]

7. Falcini F, Resti M, Azzari C, Simonini G, Veltroni M, Lionetti P. Acute febrile cholestasis as an inagural manifestation of Kawasaki's disease. Clin Exp Rheumatol 2000; 18: 779-80. 
8. Dorongspisitkul K, Gururaj VJ, Park JM, Martin CF. The prevention of coronary artery aneurrysm in Kawasaki disease: a meta-analysis on the efficacy of aspirin and immunoglobulin treatment. Pediatrics 1995; 96: 1057-61

9. Lang BA, Yeung RS, Oen KG, Malleson PN, Huber AM, Riley M et al. Corticosteroid treatment of refractory Kawasaki disease. J Rheumatol 33: 803-9.
10. Singh R, Ward C, Walton M, Persad R. Atypical Kawasaki disease and gastrointestinal manifestations. Paediatr Child Health 2007; 12: 235-7. [CrossRef]

11. Chen CJ, Huang FC, Tiao MM, Huang YH, Lin LY, Yu HR, et al. Sonographic gallbladder abnormality is associated with intravenous immunoglobulin resistance in Kawasaki disease. ScientificWorldJournal 2012; 2012: 485758. [CrossRef] 